\title{
Low-resolution spectroscopy of the Sunyaev-Zel'dovich effect and estimates of cluster parameters
}

\author{
P. de Bernardis ${ }^{1,2}$, S. Colafrancesco ${ }^{3,4}$, G. D’Alessandro ${ }^{1}$, L. Lamagna ${ }^{1,2}$, \\ P. Marchegiani ${ }^{3}$, S. Masi ${ }^{1,2}$, and A. Schillaci ${ }^{1,2}$
}

\author{
1 Dipartimento di Fisica, Università di Roma "La Sapienza", Roma, Italy \\ e-mail: paolo.debernardis@roma1.infn.it \\ 2 INFN Sezione di Roma 1, Roma, Italy \\ 3 INAF - Osservatorio Astronomico di Roma, Monte Porzio Catone, Italy \\ ${ }^{4}$ School of Physics, University of the Witwatersrand, Johannesburg Wits 2050, South Africa \\ Received 9 September 2011 / Accepted 8 November 2011
}

\begin{abstract}
Context. The Sunyaev-Zel'dovich (SZ) effect is a powerful tool for studying clusters of galaxies and cosmology. Large mm-wave telescopes are now routinely detecting and mapping the SZ effect in a number of clusters, measure their comptonisation parameter and use them as probes of the large-scale structure and evolution of the universe.

Aims. We show that estimates of the physical parameters of clusters (optical depth, plasma temperature, peculiar velocity, non-thermal components etc.) obtained from ground-based multi-band SZ photometry can be significantly biased, owing to the reduced frequency coverage, to the degeneracy between the parameters and to the presence of a number of independent components larger than the number of frequencies measured. We demonstrate that low-resolution spectroscopic measurements of the SZ effect that also cover frequencies $>270 \mathrm{GHz}$ are effective in removing the degeneracy.

Methods. We used accurate simulations of observations with lines-of-sight through clusters of galaxies with different experimental configurations (4-band photometers, 6-band photometer, multi-range differential spectrometer, full coverage spectrometers) and different intracluster plasma stratifications.

Results. We find that measurements carried out with ground-based few-band photometers are biased towards high electron temperatures and low optical depths, and require coverage of high frequency and/or independent complementary observations to produce unbiased information; a differential spectrometer that covers 4 bands with a resolution of $\sim 6 \mathrm{GHz}$ eliminates most if not all bias; full-range differential spectrometers are the ultimate resource that allows a full recovery of all parameters.
\end{abstract}

Key words. techniques: spectroscopic - galaxies: clusters: intracluster medium - cosmic background radiation

\section{Introduction}

The Sunyaev-Zel'dovich (SZ) effect (Sunyaev \& Zeldovich 1972) is the inverse Compton energisation of cosmic microwave background (CMB) photons that cross the hot plasma in clusters of galaxies. The same effect is expected in other astrophysical environments, such as the jets and lobes of giant radio-galaxies (see e.g. Colafrancesco et al. 2008).

The SZ effect is a powerful tool for studying the physics of clusters and for using them as cosmological probes (see e.g. Birkinshaw 1999; Carlstrom et al. 2002; Rephaeli et al. 2006).

Large mm-wave telescopes (Carlstrom et al. 2011; Swetz et al. 2011; Schwan et al. 2010), coupled to imaging multi-band arrays of bolometers, are now operating in excellent sites and produce a number of detections and maps of the SZ effect, discover new clusters, and establish cluster and cosmological parameters.

Meanwhile, the Planck space mission (Planck collaboration 2011a) has recently produced a shallow whole-sky survey in nine $\mathrm{cm}$ to submm bands, from which an early catalogue of massive clusters detected via the SZ effect has been extracted (Planck collaboration 2011b). The Early-SZ catalogue consists of 169 known clusters, plus 20 new discoveries, including exceptional members (Planck collaboration 2011c).

All these measurements take advantage of the extreme sensitivity of bolometers, with their excellent performance in the frequency range 90-600 GHz where the spectral signatures of the SZ effect lie.

Because all measurements integrate signals along the line of sight (LOS), we summarize below the dependence of the LOS signal on the physical parameters of the cluster. Several components should be considered:

a) A thermal component. In the non-relativistic approximation for the cluster plasma, the change of the CMB brightness $I$ in a LOS crossing the cluster is (see e.g. Birkinshaw 1999)

$\frac{\Delta I_{\mathrm{t}}}{I_{\mathrm{CMB}}}=y \frac{x^{4} \mathrm{e}^{x}}{\left(\mathrm{e}^{x}-1\right)^{2}}[x \operatorname{coth}(x / 2)-4]$,

where $I_{\mathrm{CMB}}$ is the brightness of the CMB, $x=h v / k T$ and

$y=\int_{\mathrm{LOS}} \frac{k T_{\mathrm{e}}}{m_{\mathrm{e}} c^{2}} n_{\mathrm{e}} \sigma_{T} \mathrm{~d} \ell$,

which is proportional to the integral of the pressure along the LOS. For rich clusters the optical depth is $\tau_{\mathrm{t}}=\int_{\mathrm{LOS}} n_{\mathrm{e}} \sigma_{T} \mathrm{~d} \ell \lesssim$ 0.01 and the average energy boost of $\mathrm{CMB}$ photons is $\Delta E / E \sim k T_{\mathrm{e}} / m_{\mathrm{e}} c^{2} \lesssim 1 \%$, so that a $\Delta T / T \lesssim 10^{-4}$ is expected. This is reasonably large with respect to the intrinsic CMB anisotropy, and has a characteristic spectrum, with a decrement of CMB brightness at $v \lesssim 218 \mathrm{GHz}$ and an increment of CMB brightness at $v \gtrsim 218 \mathrm{GHz}$. This peculiar 
spectrum is the key to distinguish the thermal SZ effect from competing contributions (see below). Relativistic corrections have also been computed for improved precision (see e.g. Itoh et al. 1998) and are used to characterize the physical parameters of the cluster atmospheres (see e.g. Colafrancesco et al. 2011).

b) A Doppler component, caused by the collective motion of the cluster with velocity $v$ in the CMB restframe. The spectrum of this effect is the same as the spectrum of the intrinsic CMB anisotropy, so that the two contributions cannot be separated. The amplitude of the signal is

$\frac{\Delta T}{T} \sim-\tau_{\mathrm{t}} \frac{v_{\mathrm{LOS}}}{c}$,

where $v_{\text {LOS }}$ is the projection of $v$ along the LOS; so we get

$$
\frac{\Delta I_{\mathrm{v}}}{I_{\mathrm{CMB}}} \sim-\tau_{\mathrm{t}} \frac{v_{\mathrm{LOS}}}{c} \frac{x \mathrm{e}^{x}}{\left(\mathrm{e}^{x}-1\right)}
$$

c) A non-thermal component $\Delta I_{\text {nt }}$, caused by a non-thermal population of electrons, produced by e.g. the AGNs present in the cluster, relativistic plasma in cluster cavities, shock acceleration. If dark matter in galaxy clusters consisted of neutralinos, their annihilation would produce high-energy charged particles as well. For a review, see e.g., Colafrancesco (2010), and references therein. The inverse-Compton spectrum of this component is very different from that of the thermal component, because the energy of CMB photons is boosted to frequencies much higher than the submm frequencies observable with CMB instruments (see Colafrancesco 2008, for details). This component is normally sub-dominant with respect to the thermal component: its optical depth $\tau_{\text {nt }}$ is at least 50 times less than $\tau_{\mathrm{t}}$. The parameters characterizing the spectrum, in addition to the optical depth, are the spectral index of the power-law spectrum of the energy of the electrons $\alpha$ (typically around -2.7 ), and their minimum momentum $p_{1}$, typically of the order of a few $\mathrm{MeV} / \mathrm{c}$.

d) Additional sources of signal along the same line of sight are the intrinsic anisotropy of the CMB (see point b) above)

$$
\frac{\Delta I_{\mathrm{CMBi}}}{I_{\mathrm{CMB}}}=\frac{x \mathrm{e}^{x}}{\left(\mathrm{e}^{x}-1\right)} \frac{\Delta T}{T} .
$$

Since components b) and d) have exactly the same spectrum, we will describe them in the following with the parameter $\Delta I_{\mathrm{CMB}}=\Delta I_{\mathrm{CMBi}}+\Delta I_{\mathrm{v}}$ (or the equivalent $\Delta T_{\mathrm{CMB}}$ ), which is the sum of the intrinsic anisotropy of the CMB and of the kinetic SZ effect along the line of sight.

e) The emission of dust $\Delta I_{\mathrm{d}}$ in our Galaxy and in the galaxies of the cluster. This is modelled as a thermal spectrum with temperature $T_{\mathrm{d}} \sim 20 \mathrm{~K}$ and a spectral index of emissivity $\sim-1.5$, or a superposition of several components with different $T_{\mathrm{d}}$. It is important as a contaminant at frequencies where the thermal SZ is positive.

f) The free-free and synchrotron emission $\left(\Delta I_{\mathrm{ff}}, \Delta I_{\mathrm{sy}}\right)$ from the diffuse medium in our Galaxy and from the galaxies in the cluster: this component can be important at low frequencies, where the $\mathrm{SZ}$ is negative.

According to these considerations it follows that SZ measurements promise to estimate several physical parameters of the cluster on the line of sight, provided there are more observation bands than parameters to be determined, or some of the contributions are known to be negligible.

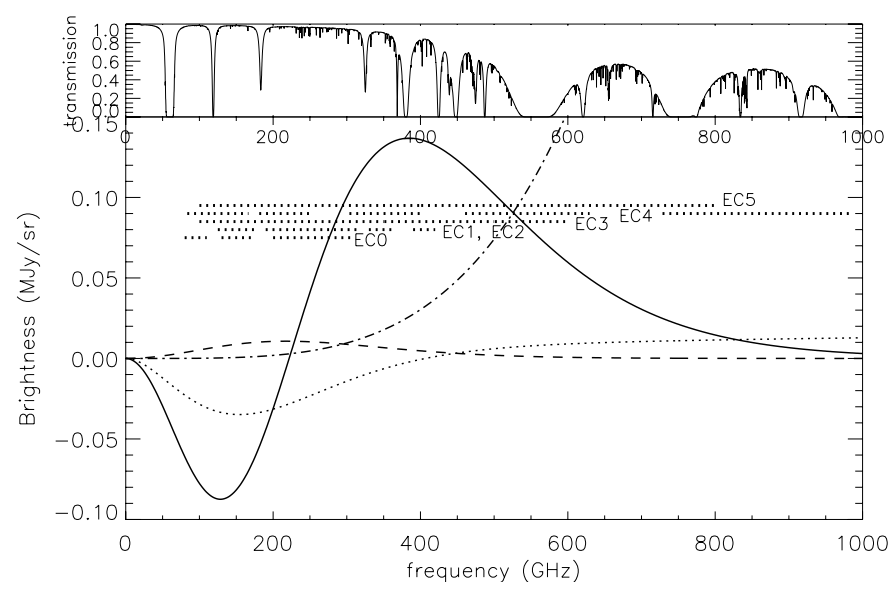

Fig. 1. Thermal SZ spectrum (continuous line in bottom panel), compared to the atmospheric transmission of a dry, cold, atmosphere (top panel, $\mathrm{PWV}=0.5 \mathrm{~mm}$ ), and to spectra of the non-thermal SZ effect (dotted line), of CMB anisotropy and kinematic SZ (dashed line), and of dust anisotropy (dot-dashed line). The parameters of the different spectra are for the benchmark case detailed in Sect. 2: $\tau_{\mathrm{t}}=5 \times 10^{-3}, T_{\mathrm{e}}=$ $8.5 \mathrm{keV}, \Delta T_{\mathrm{CMB}}=22 \mu \mathrm{K}, \tau_{\mathrm{nt}}=1 \times 10^{-4}, \alpha=-2.7, p_{1}=1.4 \mathrm{MeV} / \mathrm{c}$, $\Delta I_{\mathrm{d}}(150 \mathrm{GHz})=600 \mathrm{Jy}$. The frequency coverage of the different experiments considered in the paper is also shown as dotted horizontal lines, labelled with the experimental configuration number.

Multi-frequency measurements are therefore mandatory to separate the contributions of the different physical components, taking advantage of the characteristic spectrum of the SZ effect, which significantly departs from the spectra of the foreground and background components (see Fig. 1). The wider and more detailed the frequency coverage of the observations, the more effective the separation of the different components.

The recent results of Planck (Planck collaboration 2011b), for example, have been obtained by exploiting the excellent frequency coverage of the mission and sophisticated component separation techniques (see e.g. Leach et al. 2008).

In this paper we study how effective the various experimental configurations are in separating all the different physical components and in providing unbiased estimates of the cluster parameters (like $y, v, T_{\mathrm{e}}, \tau_{\mathrm{t}}, \tau_{\mathrm{nt}}, p_{1} \ldots$ ) and of the other parameters that describe the signals along the same line of sight $\left(T_{\mathrm{d}}, \tau_{\mathrm{d}}, I_{\mathrm{s}}, I_{\mathrm{ff}}\right.$, $\left.\Delta I_{\mathrm{CMBi}}, \ldots\right)$.

Evidently, ground-based few-band photometers cannot provide enough information to separate all physical components. Observations are hampered at high frequencies $(\gtrsim 200 \mathrm{GHz})$ by atmospheric noise (see Fig. 1): this significantly limits the coverage of the positive part of the thermal SZ spectrum, and makes the removal of parameter degeneracies much more difficult. These instruments need external information (optical, X-ray, far-IR, etc.) to produce mainly measurements of $\tau_{\mathrm{t}}$. With the addition of external data, these experiments provide invaluable information in the current exploration phase: a large database of clusters is being built, and new cluster candidates have been discovered (see e.g. Hincks et al. 2010; Marriage et al. 2010; Brodwin et al. 2011; Hand et al. 2011; Sehgal et al. 2011; Foley et al. 2011; Story et al. 2011; Williamson et al. 2011).

In principle, future space-based spectrometers can cover the full range of interesting frequencies and offer much more information: with these machines it should be possible to measure the parameters of a cluster, and use external information, when available, as a cross-check. Also, other important scientific targets of these instruments are the measurement of the $\mathrm{C}^{+}$and 
CO lines in the redshift desert and beyond for a large number of galaxies (see e.g. de Bernardis et al. 2010; Gong et al. 2012).

The main purpose of this paper is to analyse quantitatively and compare the performance of these different configurations for SZ measurements.

As samples of experimental configurations we considered a ground-based 4-band photometer; a 4-band photometer and a 4band differential spectrometer (both suitable for balloon-borne observations, and similar to the configuration of the OLIMPO experiment - see Masi et al. 2008; Conversi et al. 2010), Planck HFI (Planck HFI Core Team 2011), and a full-coverage differential spectrometer suitable for a future, dedicated space mission, like SAGACE (de Bernardis et al. 2010) or Millimetron (http://www.sron.rug.nl/millimetron).

In Sect. 2 we describe and compare the considered configurations; in Sect. 3 we describe the simulations and the analysis method; in Sect. 4 we discuss the results.

\section{Experimental configurations}

All instruments considered here work at the diffraction limit, with $A \Omega=\lambda^{2}$, to achieve the best possible angular resolution, which is needed to resolve the target cluster.

We limited our analysis to experiments using arrays of bolometers because of their superior mapping speed. Having maps of a sky patch surrounding the cluster is important to identify foreground structures and the internal structure of the cluster, if the resolution is sufficient. This choice limits the frequency coverage to $v \gtrsim 70 \mathrm{GHz}$, where bolometer arrays have a significant sensitivity advantage with respect to coherent detectors.

Few-band photometers have been traditionally matched to the $\mathrm{mm} / \mathrm{submm}$ atmospheric windows, i.e. the frequency bands $\mathrm{W} 1=[75-115] \mathrm{GHz}, \mathrm{W} 2=[125-175] \mathrm{GHz}, \mathrm{W} 3=$ [190-315] GHz, W4 = [330-365] GHz, W5 = [390-420] GHz. Operation in the higher frequency windows is significantly hampered by atmospheric noise and poor transmission, which strongly depends on the telescope site. In general ground-based photometers use narrower bands within the ranges above, while balloon-borne multi-band photometers can have wider bands and can use the higher frequency bands efficiently. Satellite instruments do not have these limitations, and the operation bands are selected based on the necessity to calibrate the instrument and to study the Galactic and extragalactic contaminating foregrounds (like the CO lines, and the continuum from interstellar dust, freefree and synchrotron emission).

For our spectroscopy we considered a Fourier Transform Spectrometer (FTS) because, at variance with dispersion spectrometers (see e.g. Bradford et al. 2003), this instrument is intrinsically imaging, a crucial requirement for studying appropriately the SZ effect in cosmic structures. Moreover, at variance with Fabry-Perot spectrometers (see e.g. Benford et al. 2003) the FTS can be used in a differential configuration where a sky field is compared to an internal reference blackbody (as in the COBE-FIRAS instrument, Mather et al. 1993; or in the Herschel-SPIRE, Griffin et al. 2007), and also in a differential configuration where two sky-fields are compared, thus rejecting most of the common mode signal from the instrument, the atmosphere, the foregrounds, and the CMB (see e.g. de Bernardis et al. 2010).

Experimental Configuration 0 (EC0) is a ground-based 4-band photometer that measures the bands W1, W2, and the two halves of W3 ([200-240] GHz and [240-310] GHz) in an excellent site like the Atacama desert, South Pole, or Dome-C. We assumed $2.7 \%, 2.5 \%, 3.5 \%, 5.0 \%$ average emissivity for the atmosphere, respectively, in the 4 bands above (corresponding to a precipitable water vapor $\sim 0.5 \mathrm{~mm}$ ), and an equivalent temperature of the atmosphere of $240 \mathrm{~K}$. Only photon noise was considered, assuming that the telescope is used in a differential configuration, where most of the turbulence is subtracted out as a common mode signal.

Experimental Configuration 1 (EC1) is a balloon-borne 4-band photometer that measures bands W2, W3, W4, and W5. Here the background is limited by the temperature $(\sim 230 \mathrm{~K})$ and emissivity of the telescope mirrors and of the cryostat window. Again a differential configuration was considered.

Experimental Configuration 2 (EC2) is a balloon-borne 4-band differential spectrometer that measures the lowresolution $(\Delta v=6 \mathrm{GHz})$ spectra in four ranges coincident with W2, W3, W4, and W5. Here the background is limited by the temperature and emissivity of the telescope, of the interferometer mirrors, and of the cryostat window, all at room temperature $(230 \mathrm{~K})$. Note that in this (and in the following) photon-noise limited configurations, that use Fourier transform spectrometers, the error on each spectral bin scales as the inverse of the spectral resolution $\Delta v$ (see Sect. 3).

Experimental Configuration 3 (EC3) is an Earth-orbit (EO) satellite with a telescope radiatively cooled at $80 \mathrm{~K}$, which measures spectra in four consecutive ranges $b 0=[100,200] \mathrm{GHz}$, $b 1=[201,350] \mathrm{GHz}, b 2=[351,500] \mathrm{GHz}, b 3=[501,600] \mathrm{GHz}$ with $6 \mathrm{GHz}$ resolution. Here the background is limited by the temperature and emissivity of the cold telescope and of the cryostat window (total emissivity $\epsilon \sim 0.01$ ), while the interferometer mirrors are kept at $<4 \mathrm{~K}$ and do not contribute to the photon noise. The spectral coverage has been divided in the four ranges observed simultaneously by independent detector arrays in order to limit the radiative background on each array.

Experimental Configuration 4 (EC4) is a 6-band photometer similar to Planck-HFI, which operates in the Lagrangian point L2 of the Sun-Earth system, with the telescope radiatively cooled at $45 \mathrm{~K}$, measuring simultaneously in the bands $b 0=[83.5,116.5] \mathrm{GHz}, b 1=[119.4,166.6] \mathrm{GHz}, b 2=[181.2$, $252.8] \mathrm{GHz}, b 3=[303.6,402.4] \mathrm{GHz}, b 4=[460.5,629.5] \mathrm{GHz}$, $b 5=[728.5,985.6] \mathrm{GHz}$. Here the background is limited by the temperature and emissivity of the cold telescope (total emissivity $\epsilon \sim 0.005)$.

Experimental Configuration 5 (EC5) is a 4-range differential interferometer that operates in deep space (L2), with an actively cooled telescope $(T \lesssim 6 \mathrm{~K})$, in the bands $b 0=[100,200] \mathrm{GHz}$, $b 1=[201,400] \mathrm{GHz}, b 2=[401,600] \mathrm{GHz}, b 3=[601,800] \mathrm{GHz}$ with $6 \mathrm{GHz}$ resolution. Here the background is limited by natural radiation sources (Galactic, extragalactic, $\mathrm{CMB}$ ): this allows a wider coverage (including high frequencies) without the risk of a high background on the detectors.

For a summary see Fig. 1, where the coverage of the different configurations is compared to the SZ and foreground spectra.

\section{Simulation of line-of-sight observations}

In order to evaluate the performance of different experimental configurations, we have considered the following benchmark situation: the observation of a line of sight that crosses a rich cluster of galaxies, with $\tau_{\mathrm{t}}=0.005, T_{\mathrm{e}}=5 \mathrm{keV}, \Delta T_{\mathrm{CMB}}=22 \mu \mathrm{K}$ (corresponding to $v=480 \mathrm{~km} \mathrm{~s}^{-1}$, if the intrinsic anisotropy of the CMB along the line of sight is negligible), $\tau_{\mathrm{nt}}=0.0001$, $\left.\alpha=-2.7, p_{1}=2.75 \times 511 \mathrm{keV} / \mathrm{c}\right)$.

We assumed that the angular resolution of all channels of all experimental configurations is sufficient to resolve the source. Otherwise we would have needed to take into account dilution 
and shape factors: in this LOS approach we avoided these complications. We believe that this approach is adequate for the purpose of this paper, which is to compare the performance of different experimental configurations.

The integration time on the same line of sight is assumed to be $3 \mathrm{~h}$ for all cases but EC4. For EC4 (a space-borne whole-sky survey à-la-Planck) the integration time on the considered sky pixel is $30 \mathrm{~s}$.

The signal power on the detectors was computed as

$S(v)=A \Omega E(v)\left(1-\epsilon_{\mathrm{m}}(v)\right)\left[\Delta I_{\mathrm{t}}+\Delta I_{\mathrm{v}}+\Delta I_{\mathrm{CMB}}+\Delta I_{\mathrm{nt}}+\Delta I_{\mathrm{d}}\right]$,

where $A$ is the collecting area, $\Omega$ is the solid angle sampled by each detector, $E(v)$ is the efficiency of the detection system, $\epsilon_{\mathrm{m}}(v)$ is the total emissivity of the optical system at room temperature and of the atmosphere in the measurement band.

We have neglected $\Delta I_{\text {ff }}$ and $\Delta I_{\text {sy }}$ because they are negligible with respect to $\Delta I_{\mathrm{d}}$ in the frequency range and for the observations at high galactic latitudes of interest here. We modelled typical Galactic cirrus anisotropy at the angular scale of a cluster (a few arcmin) as $\Delta I_{\mathrm{d}}(v)=A\left(v / v_{\mathrm{o}}\right)^{4}$ with $A=600 \mathrm{Jy} / \mathrm{sr}$ and $v_{\mathrm{o}}=150 \mathrm{GHz}$ : a value typical of very clean high-latitude regions (see e.g. Masi et al. 2006).

We assumed that the detector array is optimized to be limited by the photon noise of the radiative background that is produced by the instrument and the atmosphere (if present). Cryogenic bolometers reach this performance level even in the extremely low background achievable in space, if properly designed (see e.g. Holmes et al. 2008).

The background power on the detectors was computed as

$B(v)=A \Omega E(v)\left[\epsilon_{\mathrm{m}}(v) \frac{2 h v^{3}}{c^{2}} \frac{1}{\mathrm{e}^{x_{\mathrm{m}}}-1}+\left[1-\epsilon_{\mathrm{m}}(v)\right] \frac{2 h v^{3}}{c^{2}} \frac{1}{\mathrm{e}^{x}-1}\right]$,

where $x_{\mathrm{m}}=h v / k T_{\mathrm{m}}, T_{\mathrm{m}}$ is the temperature of the optical system and of the atmosphere, $x=h v / k T, T$ is the temperature of the cosmic microwave background.

The fluctuations of the background were computed as

$$
\begin{aligned}
& N E P_{\mathrm{ph}}^{2}=N E P_{\mathrm{m}}^{2}+N E P_{\mathrm{CMB}}^{2} \\
& N E P_{\mathrm{m}}^{2}=A \Omega \frac{4 k^{5}}{c^{2} h^{3}} T_{\mathrm{m}}^{5} E(v) \epsilon_{\mathrm{m}}(v) \frac{x_{\mathrm{m}}^{4}\left[\mathrm{e}^{x_{\mathrm{m}}}-1+E(v) \epsilon_{\mathrm{m}}(v)\right]}{\left(\mathrm{e}^{x_{\mathrm{m}}}-1\right)^{2}} \\
& N E P_{\mathrm{CMB}}^{2}= \\
& A \Omega \frac{4 k^{5}}{c^{2} h^{3}} T^{5} E(v)\left(1-\epsilon_{\mathrm{m}}(v)\right) \frac{x^{4}\left[\mathrm{e}^{x}-1+E(v)\left(1-\epsilon_{\mathrm{m}}(v)\right)\right]}{\left(\mathrm{e}^{x}-1\right)^{2}} .
\end{aligned}
$$

For the photometric measurements we integrated Eqs. (6) and (8) over the detection bandwidth $B W$ to obtain the error on the signal for each band:

$\sigma_{\text {phot }}^{2}=\frac{\int_{\mathrm{BW}} N E P_{\mathrm{ph}}^{2} \mathrm{~d} v}{2 t}$,

where $t$ is the total integration time.

In the case of spectroscopic measurements, the error on the measurement of each spectral bin was computed as follows. In the FTS the input power is splitted in the two arms of the interferometer, and a variable delay is introduced in one of the two beams, before recombining them on the detector. The variable delay is introduced by a moving mirror, which can be offset by $x$ with respect to the corresponding steady mirror in the other arm of the interferometer. In this way a $2 c x$ delay is introduced. The power measured in position $x$ of the moving mirror is $P(x)$ (the interferogram), and the spectrum is estimated as the Fourier transform of the interferogram:

$$
\begin{aligned}
S(\sigma) & =\int_{-x_{\max }}^{x_{\max }}(P(x)-\langle P\rangle) \cos (4 \pi \sigma x) \mathrm{d} x \\
& =2 \int_{0}^{x_{\max }}(P(x)-\langle P\rangle) \cos (4 \pi \sigma x) \mathrm{d} x,
\end{aligned}
$$

where $\sigma$ is the wavenumber $\left(\mathrm{in}^{-1}\right.$ ). The spectral resolution of the measurement is

$$
\Delta \sigma=1.22 /\left(2 x_{\max }\right)
$$

(see e.g. Chantry 1971). In a real instrument the interferogram is sampled at the positions $x_{i}=i \Delta x,(i=1 \ldots N)$, with $N=x_{\max } / \Delta x$. The integral is then estimated as a discrete sum

$$
\begin{aligned}
S\left(\sigma_{j}\right) & =2 \sum_{i=1}^{N}\left(P\left(x_{i}\right)-\langle P\rangle\right) \cos \left(4 \pi \sigma_{j} x_{i}\right) \Delta x \\
& =1.22 \sum_{i=1}^{N}\left(P\left(x_{i}\right)-\langle P\rangle\right) \cos \left(4 \pi \sigma_{j} x_{i}\right) /(N \Delta \sigma) .
\end{aligned}
$$

Since each sample of the interferogram has an integration time $T / N$, and photons from the whole observed frequency bandwidth contribute to the noise, the error in the measurement is therefore

$\sigma_{\mathrm{P}}=\frac{\sqrt{\int_{\mathrm{BW}} N E P_{\mathrm{ph}}^{2} \mathrm{~d} v}}{\sqrt{2 t / N}}$

The error in the measurement of each spectral bin is thus

$$
\begin{aligned}
\sigma_{\mathrm{S}}^{2} & =1.22^{2} \sum_{i=1}^{N} \sigma_{P}^{2} \cos ^{2}\left(4 \pi \sigma x_{i}\right) /(N \Delta \sigma)^{2} \\
& =1.22^{2} \frac{\sigma_{P}^{2}}{N \Delta \sigma^{2}} \sum_{i=1}^{N} \frac{\cos ^{2}\left(4 \pi \sigma x_{i}\right)}{N} \sim 0.74 \frac{\sigma_{P}^{2}}{N \Delta \sigma^{2}}
\end{aligned}
$$

or

$\sigma_{\mathrm{S}}=0.61 \frac{\sqrt{\int_{\mathrm{BW}} N E P_{\mathrm{ph}}^{2} \mathrm{~d} v}}{\Delta \sigma \sqrt{t}}=0.61 \frac{\sqrt{\int_{\mathrm{BW}} N E P_{\mathrm{ph}}^{2} \mathrm{~d} v}}{c \Delta v \sqrt{t}}$.

For each experimental configuration (EC*) we simulated 3000 measurements, using Eq. (6), and adding an error term extracted from a Gaussian distribution with zero average and standard deviation derived from Eqs. (11) and (17).

Typical simulated spectral measurements are reported in Fig. 2.

\section{Results and discussion}

We fitted each simulated measurement using Eq. (6). In Table 1 we report the averages of the best-fit parameters with their standard deviation.

While giving a general idea of the relative efficiency of the different configurations, the results reported in Table 1 can be misleading in the details, since the distributions of the best-fit parameters are not Gaussian (nor symmetrical), and there are significant correlations between the parameters. This is evident from the joint likelihood contours plotted in Figs. 3, 5-7, 9-11.

For EC0 (4-band ground-based photometer), where only four independent data-sets are available for each measurement, we 
P. de Bernardis et al.: Cluster parameters with SZ
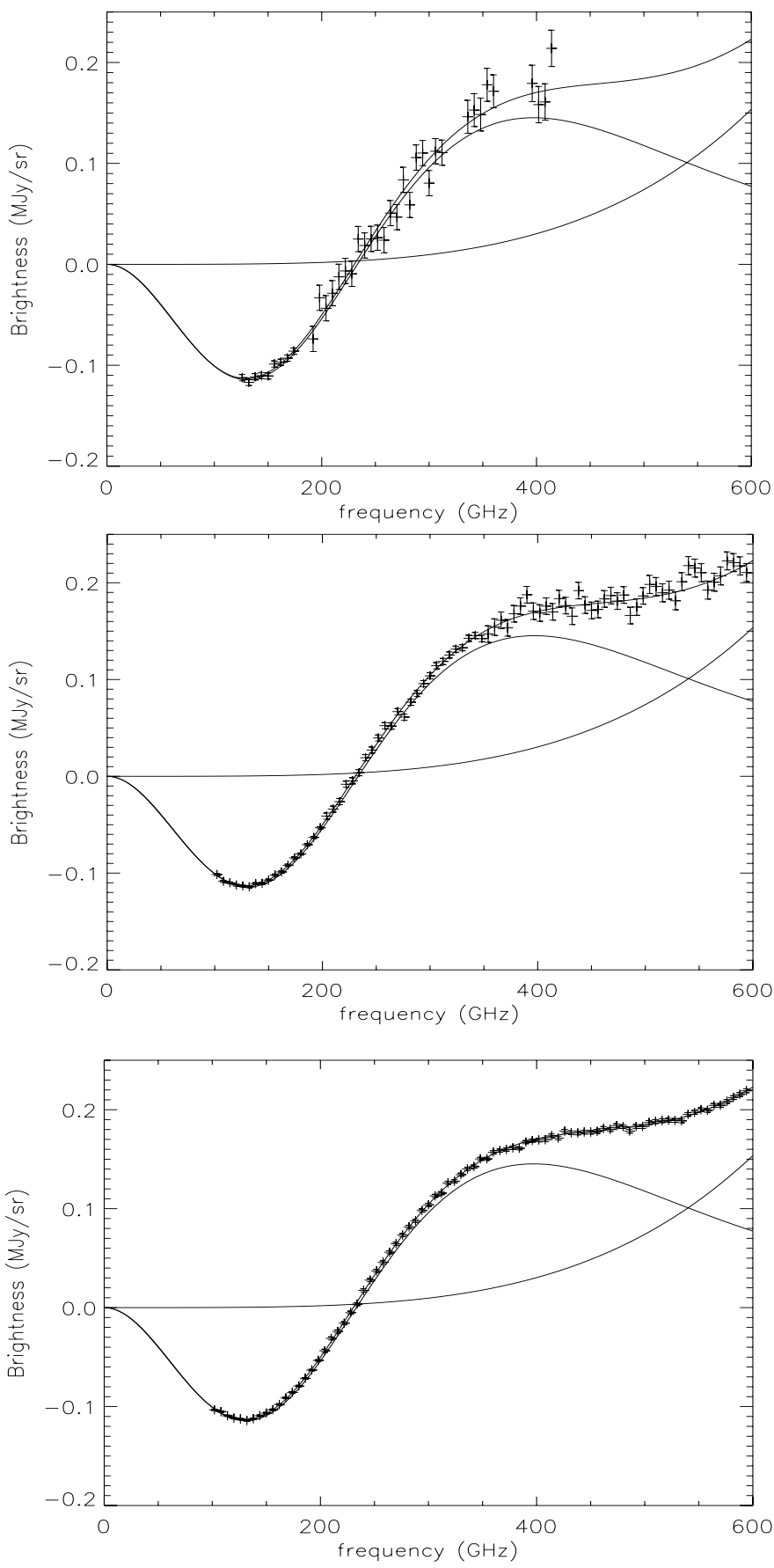

Fig. 2. From top to bottom: simulated data sets for the spectroscopic configurations EC2 (differential FTS on a stratospheric balloon, room temperature), EC3 (differential cold FTS on an earth-orbit satellite, with room temperature telescope), EC5 (differential cold FTS on a L2-orbit satellite, with cold telescope). The best-fit line through the data points is from Eq. (6). The other two lines are the thermal plus non-thermal $\mathrm{SZ}$, and the dust anisotropy.

tried to fit either three parameters $\left(\tau_{\mathrm{t}}, T, \Delta I_{\mathrm{d}}\right)$ or four parameters $\left(\tau_{\mathrm{t}}, T_{\mathrm{e}}, \Delta I_{\mathrm{d}}\right.$ and $\left.\Delta T_{\mathrm{CMB}}\right)$, adding a fictitious data point with zero brightness at zero frequency. The results are dominated by the degeneracy between $T_{\mathrm{e}}$ and $\tau_{\mathrm{t}}$, evident from Eqs. (1) and (2): without relativistic corrections, the thermal SZ depends on the product of electron density and electron temperature. For this reason a decrease of, say, a factor 2 of $\tau_{\mathrm{t}}$ is almost perfectly compensated for by an increase of a factor 2 of $T_{\mathrm{e}}$. The only way
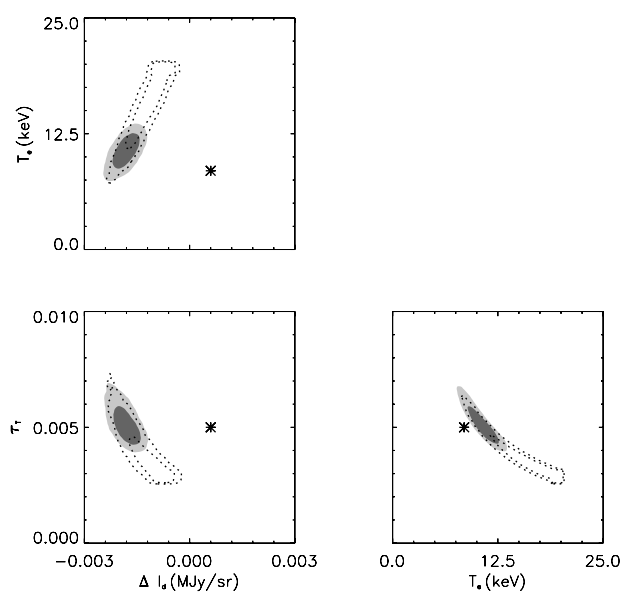

Fig. 3. Joint likelihood contours ( $95.6 \%$ and $68.7 \%$ ) for couples of bestfit parameters for the experimental configuration EC0 (ground based observations with a 4-band photometer) where the data are analysed by fitting the three parameters $\tau_{\mathrm{t}}, T_{\mathrm{e}}, \Delta I_{\mathrm{d}}$. The filled contours are for a Gaussian prior on $T_{\mathrm{e}}$ with $\sigma=3 \mathrm{keV}$; the dashed contours are for a Gaussian prior with $\sigma=8 \mathrm{keV}$. The * symbols mark the input values of parameters. In this case a significant bias for all parameters is evident. The anti-correlation between $\tau_{\mathrm{t}}$ and $T_{\mathrm{e}}$ is also evident.

to break the degeneracy is through the relativistic corrections, which, however, are very small: their effect is negligible with respect to the typical uncertainties of ground-based measurements. For this reason we had to use a prior on $T_{\mathrm{e}}$, assuming that the information is obtained through independent X-ray measurements of the specific brightness of the cluster along the same line of sight. We tried a very weak prior, with a Gaussian distribution centred on the true value and a standard deviation of $8 \mathrm{keV}$ : otherwise the best fit would converge to non-physical values for $T_{\mathrm{e}}$. The bias is only mitigated by the introduction of this prior. In the 3 -parameter case the best fits converge on values of the parameters that are not very close (in units of their standard deviation) to the input values, and the typical $\chi^{2}$ is high, confirming that the effect of the measurement error is negligible with respect to the effect of the parameter degeneracies and to the necessity of neglecting the non-thermal component in the fit.

In Fig. 3 we plot the joint likelihood contours for couples of parameters in the 3-parameter fits. The comparison with Fig. 5 shows that part but not all of the bias depends on the presence of the non-thermal component: indeed the bias changes with $\tau_{\mathrm{nt}}=$ 0 , but is still present.

If a 4-parameter fit is used, the bias is significantly reduced, and does not depend anymore on the presence of the non-thermal component. However, this comes at the cost of wider confidence areas, as evident from Fig. 6.

The contamination of dust is significant and degenerates to some extent also with $\Delta T_{\mathrm{CMB}}$ : in the 3-parameter fit, where $\Delta T_{\mathrm{CMB}}$ is not fitted, the best-fit for $\Delta I_{\mathrm{d}}$ is far from the input value, and this probably contributes to the tension between the best-fit value of $T_{\mathrm{e}}$ and its input value. We believe that this is the result of a degeneracy of parameters combined with the poor coverage of high frequencies in ground-based experiments. In the 4-parameter fits the $\chi^{2}$ improves, but the best fits are still biased, and only with the $3 \mathrm{keV}$ prior the estimates of all parameters are consistent with the input values (see Fig. 6).

For EC1 (4-bands balloon-borne photometer) the coverage of frequencies higher than $300 \mathrm{GHz}$ helps in removing the degeneracy (see Fig. 7). If we use the very weak prior on $T_{\mathrm{e}}$ (Gaussian with standard deviation $8 \mathrm{keV}$ ), the best-fit cluster 

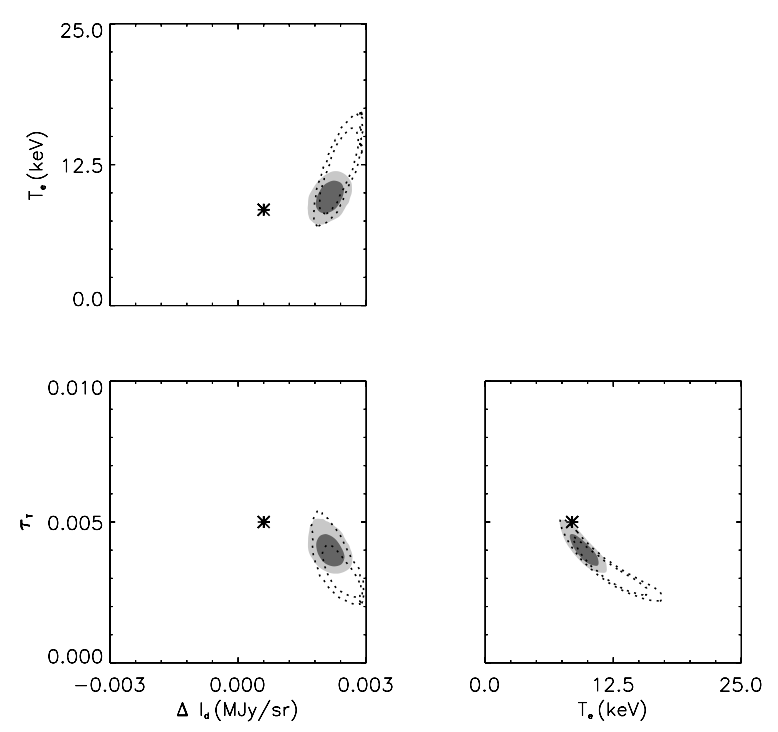

Fig. 4. Same as Fig. 3 but with $\tau_{\mathrm{nt}}=0$. The bias changes, but does not vanish.
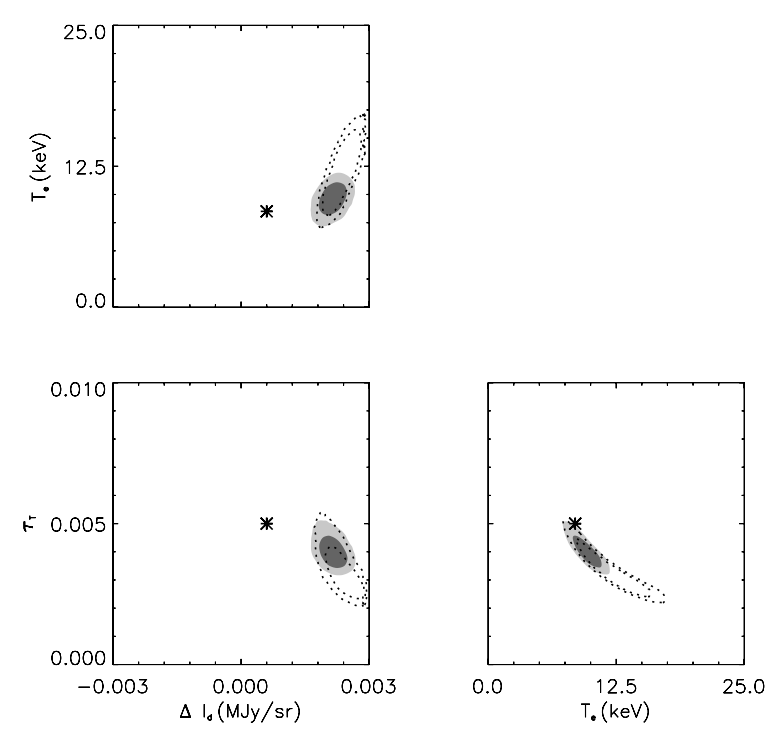

Fig. 5. Same as Fig. 3 but with $\tau_{\mathrm{nt}}=0$. The bias changes, but does not vanish.

parameters are already close to the input values, even if the error (completely dominated by the degeneracy) is relatively large. The situation improves, of course, if the standard deviation on the prior is reduced to $3 \mathrm{keV}$. $\Delta T_{\mathrm{CMB}}$ is better constrained, but is biased low. While performing significantly better than EC0, this configuration is limited by radiation noise in the high-frequency bands, which can be removed only by cooling the telescope or/and adding spectroscopic capabilities (see below).

In Fig. 8 we plot the histograms of the best-fit $T_{\mathrm{e}} \times \tau_{\mathrm{t}}$ normalized to the input of the simulation. It is evident how the bias is reduced passing from ECO with three parameters to ECO with four parameters to EC1, which shows how important the coverage of high frequencies is, which are difficult to observe from the ground.

For EC4, where six independent data-sets are available for each measurement, we tried to fit four parameters $\left(\tau_{\mathrm{t}}, T_{\mathrm{e}}, \Delta I_{\mathrm{d}}\right.$ and $\Delta T_{\mathrm{CMB}}$, see Fig. 9 ) and also six parameters (including $\tau_{\text {nt }}$ and $p_{1}$, see Fig. 10), always adding the fictitious zero frequency zero brightness data point. The good coverage of high
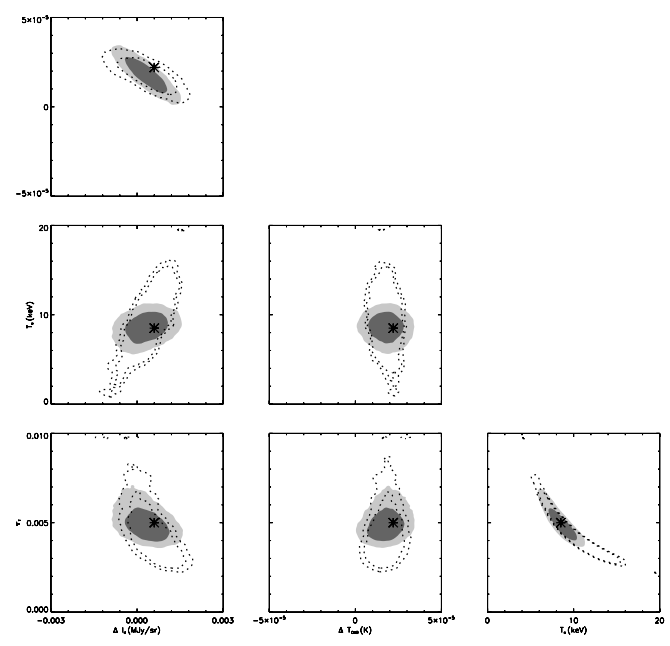

Fig. 6. Same as Fig. 3 when the data are analysed by fitting four parameters $\left(\tau_{\mathrm{t}}, T_{\mathrm{e}}, \Delta I_{\mathrm{d}}\right.$ and $\left.\Delta T_{\mathrm{CMB}}\right)$. The bias is reduced, but the anti-correlation between $\tau_{\mathrm{t}}$ and $T_{\mathrm{e}}$ is still evident and the confidence contours are wider.
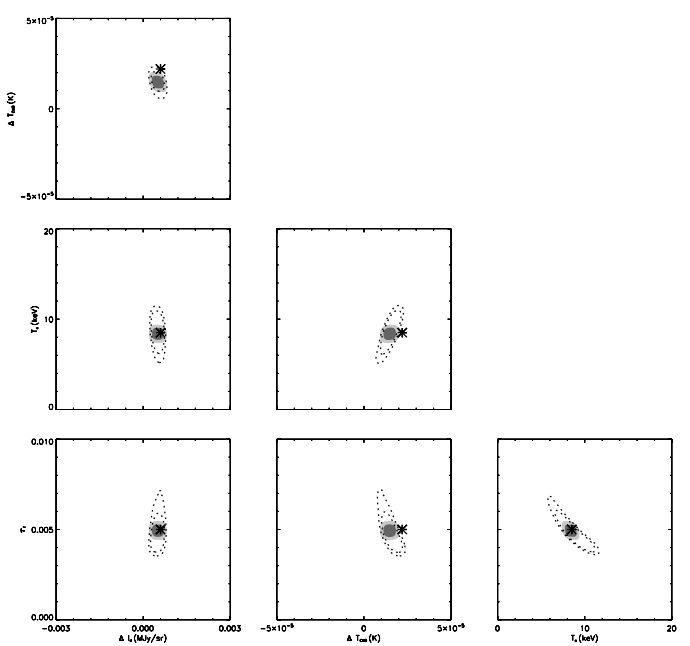

Fig. 7. Joint likelihood contours $(95.6 \%$ and $68.7 \%)$ for couples of bestfit parameters for the experimental configuration EC1 (balloon-borne observations with a 4-band photometer, extending the coverage to high frequencies not observable from the ground) where the data are analysed by fitting four parameters $\left(\tau_{\mathrm{t}}, T_{\mathrm{e}}, \Delta I_{\mathrm{d}}, \Delta T_{\mathrm{CMB}}\right)$. The filled contours are for a Gaussian prior on $T_{\mathrm{e}}$ with $\sigma=3 \mathrm{keV}$; the dashed contours are for a Gaussian prior with $\sigma=8 \mathrm{keV}$. The * symbols mark the input values of parameters.

frequencies and low photon noise owing to the low radiative background results in very good performance in terms of statistical errors. Again, the presence of the non-thermal component produces a bias in the determination of the other parameters for the 4-parameter fit. In the 6-parameter fits, the non thermal parameters are basically not constrained (with a bimodal distribution of the best fit $p_{1}$ ), but the other parameters are well constrained and unbiased. The effects of parameters degeneracies are still evident, however, and it is difficult to estimate $\Delta T_{\mathrm{CMB}}$ because it tends to be biased low in the 4-parameter fit, while is not well constrained in the 6-parameter fits (see also Table 1).

The space spectrometers EC2, EC3, and EC5, featuring wide frequency coverage and low radiative background noise, perform better, and allow an unbiased recovery of six parameters with increasing accuracy. If we compare the parameter space volume constrained by the different configurations, we find that 
P. de Bernardis et al.: Cluster parameters with SZ

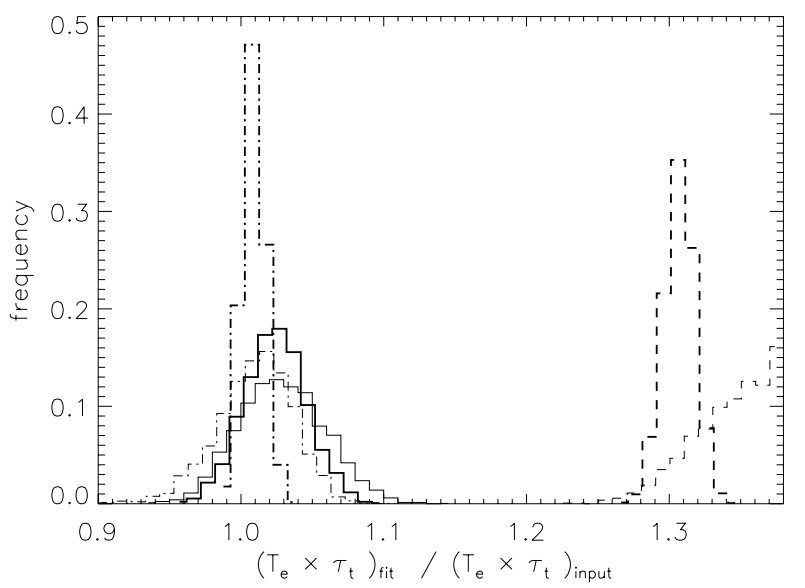

Fig. 8. Histograms of the best-fit $T_{\mathrm{e}} \times \tau_{\mathrm{t}}$ normalized to the input of the simulation, for EC0 with three parameter fits (dashed lines), EC0 with four parameter fits (continuous lines) and EC1 with four parameter fit (dot-dashed lines). Thin lines are for a Gaussian prior on $T_{\mathrm{e}}$ centred on the input value of $T_{\mathrm{e}}$ and with a standard deviation of $8 \mathrm{keV}$; thick lines are for a standard deviation of $3 \mathrm{keV}$.
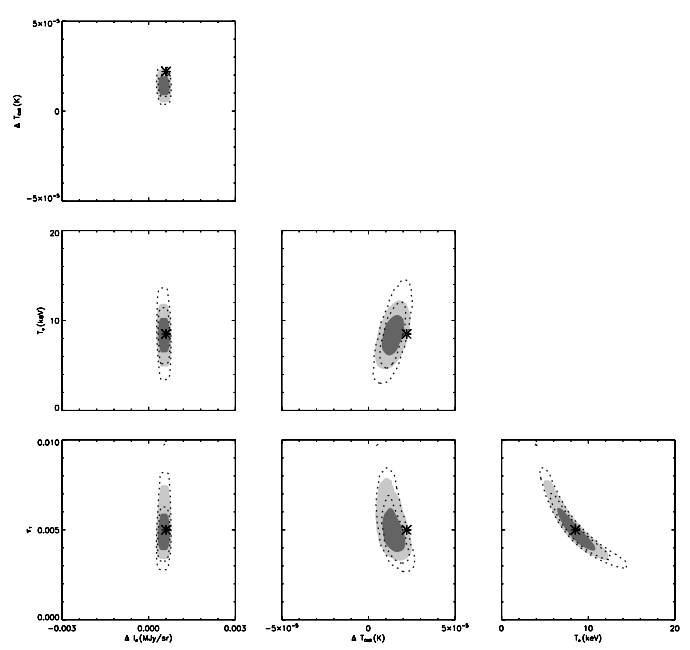

Fig. 9. Joint likelihood contours (95.6\% and 68.7\%) for couples of bestfit parameters for the experimental configuration EC4 (6-band cryogenic photometer operating in L2) where the data are analysed by fitting four parameters $\left(\tau_{\mathrm{t}}, T_{\mathrm{e}}, \Delta I_{\mathrm{d}}, \Delta T_{\mathrm{CMB}}\right)$. The filled contours are for a Gaussian prior on $T_{\mathrm{e}}$ with $\sigma=3 \mathrm{keV}$; the dashed contours are for a Gaussian prior with $\sigma=8 \mathrm{keV}$. The * symbols mark the input values of parameters.

with respect to EC2, EC3 reduces the volume by a factor 4.6, and EC5 by a factor 27 (with the $8 \mathrm{keV}$ prior).

The balloon-borne warm spectrometer EC2 is still limited at high frequencies by radiative background fluctuations. For this reason $\Delta T_{\mathrm{CMB}}$ is basically not constrained (see Fig. 11). However, the other parameters are unbiased and well constrained even with the very weak prior on $T_{\mathrm{e}}$. An experiment like OLIMPO, which combines photometric measurements (as in EC1) and spectroscopic measurements (as EC2), performing both during the same flight, can use the first measurement to optimally constrain $\Delta I_{\mathrm{d}}$ and $\Delta T_{\mathrm{CMB}}$, and the second to better constrain $\tau_{\mathrm{t}}$ and $T_{\mathrm{e}}$. Note that even $\tau_{\mathrm{nt}}$ is close to be detected (and can be detected with an integration time longer than the $3 \mathrm{~h}$ considered here) and $p_{1}$ is also constrained (still with a bimodal distribution). Finally note that the spectroscopic capabilities allow the user to remove the contamination of Galactic $\mathrm{CO}$

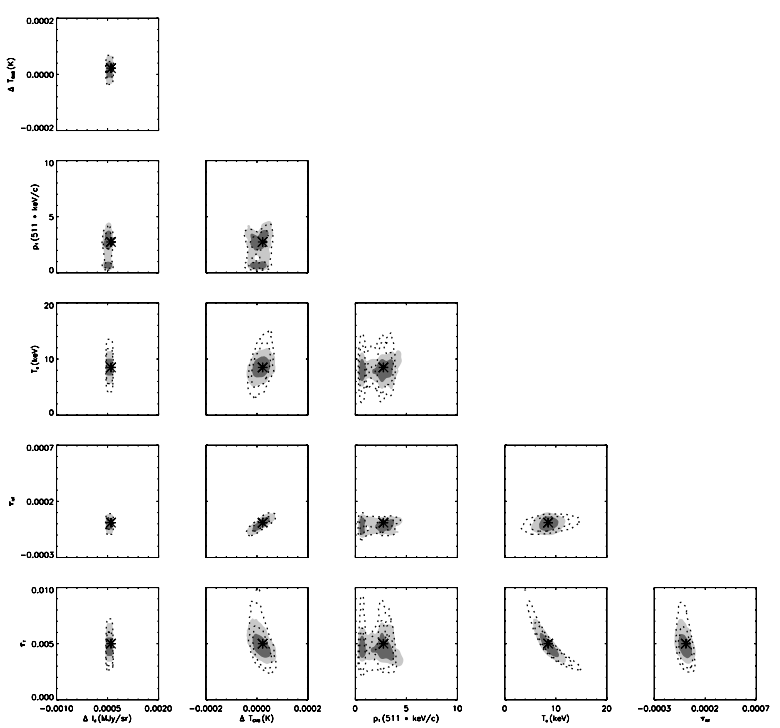

Fig. 10. Same as Fig. 9 (configuration EC4) when the data are analysed by fitting six parameters $\left(\tau_{\mathrm{t}}, T_{\mathrm{e}}, \Delta I_{\mathrm{d}}, \Delta T_{\mathrm{CMB}}, \tau_{\mathrm{nt}}, p_{1}\right)$.

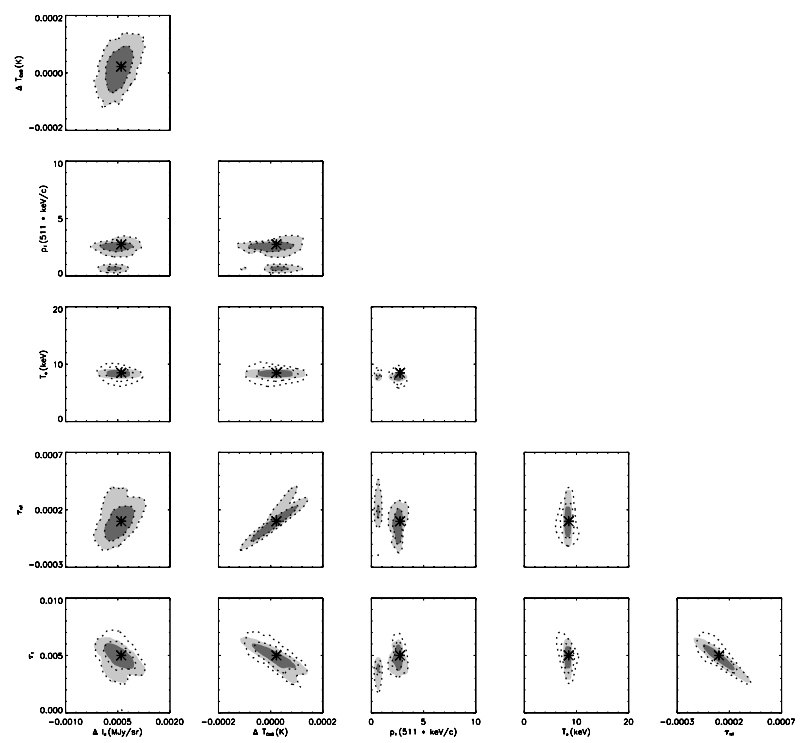

Fig. 11. Joint likelihood contours $(95.6 \%$ and $68.7 \%)$ for couples of best-fit parameters for the experimental configuration EC2 (4-range warm spectrometer on a balloon platform). The filled contours are for a Gaussian prior on $T_{\mathrm{e}}$ with $\sigma=3 \mathrm{keV}$; the dashed contours are for a Gaussian prior with $\sigma=8 \mathrm{keV}$. The * symbols mark the input values of parameters.

and other cooling lines within the photometric bands unambiguously.

The low-Earth-orbit case EC3 improves over the balloon spectrometer EC2. Cooling the spectrometer results in a significant unbiased measurement of all six parameters, including the elusive $\Delta T_{\mathrm{CMB}}$ (see Fig. 12). Because the noise is reduced, the effect of degeneracies is more evident. The $3 \mathrm{keV}$ prior is needed to fully exploit the potential of this configuration.

Experimental configuration EC5, where both the spectrometer and the telescope are cold, results in an improved precision of the determination of all parameters, and little sensitivity to the prior on $T_{\mathrm{e}}$ (see Fig. 13 and Table 1).

We have investigated the possibility of extending the frequency coverage of EC5 up to $1 \mathrm{THz}$. Keeping the same 
Table 1. Results of simulations.

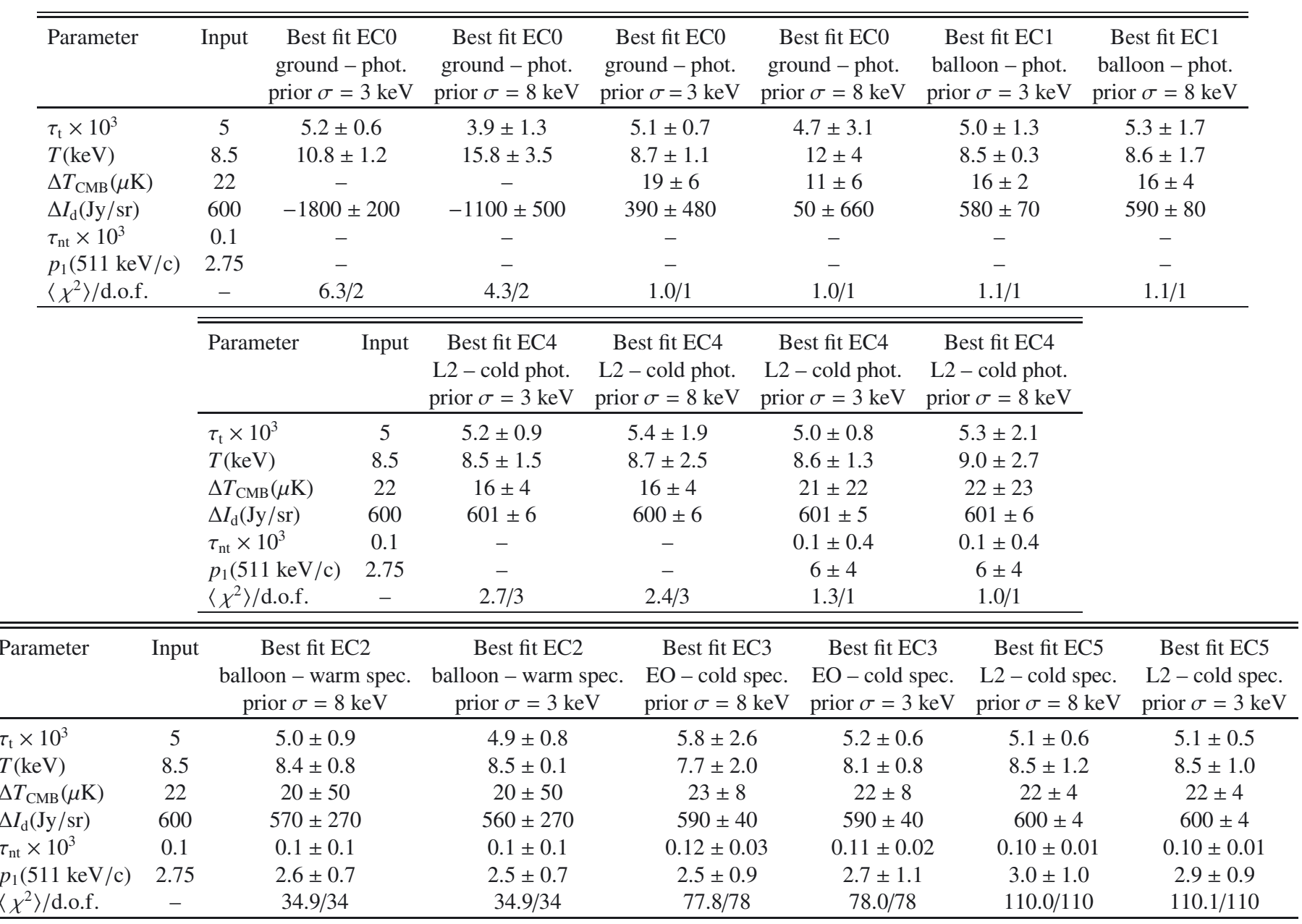

Notes. For each experimental configuration (EC*) we simulated 3000 measurements and fitted each simulated measurement using Eq. (6). We report the averages of the best-fit parameters with their standard deviation. In the top table we summarize the results for 4-band photometers for both weak and medium prior for $T_{\mathrm{e}}$; in the middle table we report the results for a 6-band photometer (Planck-HFI bands). In the bottom table we report the results for low-resolution $(\Delta v=6 \mathrm{GHz})$ spectrometers with increasing mission complexity (balloon, Earth orbit, orbit around the Sun-Earth Lagrangian point L2).

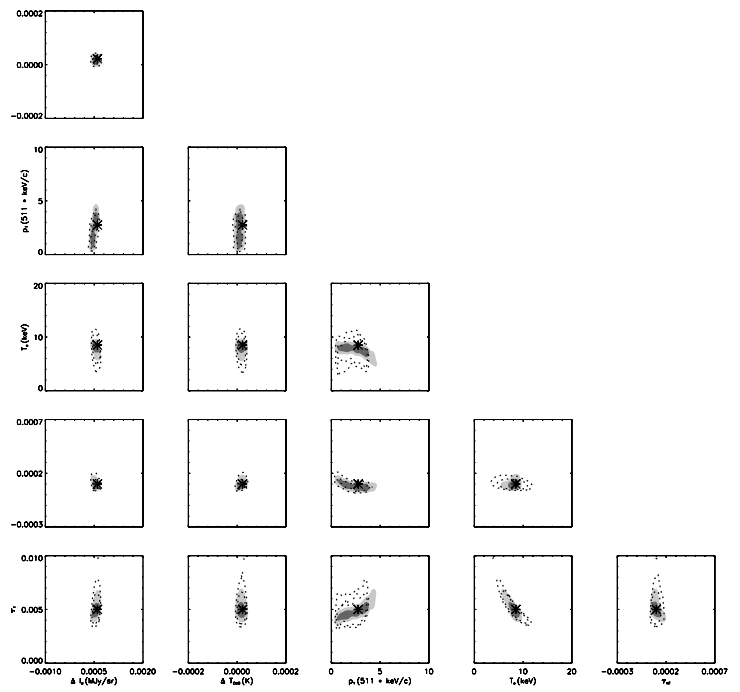

Fig. 12. Same as Fig. 11 for the experimental configuration EC3 (cold spectrometer on an Earth-orbit satellite with radiatively cooled $(80 \mathrm{~K})$ telescope).

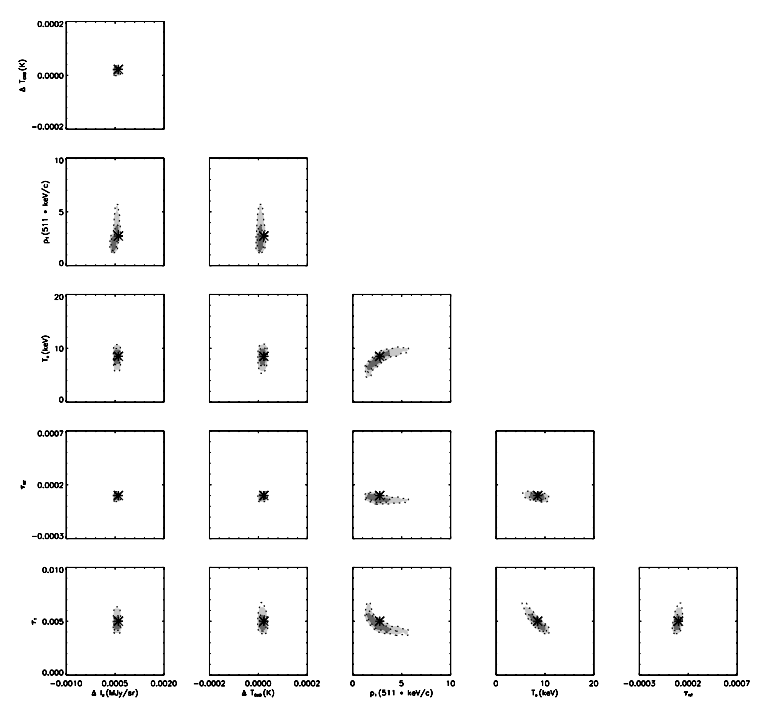

Fig. 13. Same as Fig. 11 for the experimental configuration EC5 (cold spectrometer on a satellite in L2, with actively cooled (4 K) telescope). 
simplified model for dust, we found an improvement of about a factor 2 in the uncertainties of all parameters but $\Delta T_{\mathrm{CMB}}$ (which does not improve) and $\Delta I_{\mathrm{d}}$ (which improves by a factor 5). However, our simplistic model with a single dust population probably starts to be insufficient at these high frequencies. There are, however, other drivers to extend the frequency coverage of such an ambitious experiment as high as possible: the study of interstellar atoms and molecules and the study of galaxies in the redshift desert would benefit significantly from such an extension.

\section{Conclusions}

With this simulation-based study we confirmed that few-band photometric measurements of the SZ effect can be significantly biased, and that most of the bias is removed by adding bands and covering high frequencies $(v \gtrsim 240 \mathrm{GHz})$. This requires the use of space-borne experiments. Low-resolution spectroscopic measurements of the SZ are extremely promising. An exploratory experiment, featuring a four-band photometer on a balloon and a plug-in room-temperature FTS (configurations EC1 and EC2, modelled on the OLIMPO experiment Masi et al. 2008) is required to confirm that the expectations estimated here can really be achieved. In other words, we relied here on the differential properties of FTS instruments: we need to demonstrate experimentally that systematic effects are negligible even with the extreme requirements of SZ measurements. OLIMPO, in the dual photometric-spectroscopic configuration, is a perfect demonstrator, and is expected to produce exciting improvements on the physics of cosmic structures derived from the SZ effect. Satellite missions can accommodate a cryogenic FTS (a large cryostat is required anyway to provide the required hold time), reducing the instrumental background at high frequency. A Molniya-orbit mission (like the proposed SAGACE de Bernardis et al. 2010) represents a cost-effective solution, able to provide an extensive catalogue of unbiased SZ measurements in various cosmic structures (e.g. galaxy clusters and groups, radiogalaxy lobes, gaseous halos of galaxies). A cold telescope in L2 (like the proposed Spectrum-M - millimetron mission) would be limited only by the intrinsic degeneracy of the parameters, and would open new horizons in SZ science, allowing a precise determination of both the thermal and non-thermal components of the plasma in the cluster.
Acknowledgements. This work has been supported by Italian Space Agency contracts "Millimetron" and "OLIMPO" and by PRIN 2009 "Mm and submm spectroscopy for high-resolution studies of primeval galaxies and clusters of galaxies" of the Italian Ministero dell'Istruzione, dell'Università e della Ricerca. S.C. acknowledges support by the South African Research Chairs Initiative of the Department of Science and Technology and National Research Foundation and by the Square Kilometre Array (SKA).

\section{References}

Benford, D. J., Moseley, S. H., Stacey, G. J., et al. 2003, Proc. SPIE, 4857, 105 Birkinshaw, M. 1999, Phys. Rept., 310, 97

Bradford, C. M., Naylor, B. J., Zmuidzinas, J., et al. 2003, Proc. SPIE, 4850, 1137

Brodwin, M., Ruel, J., Ade, P. A. R., et al. 2010, ApJ, 721, 90

Carlstrom, J. E., Holder, G. P., \& Reese, E. D. 2002, ARA\&A, 40, 643

Carlstrom, J. E., Ade, P. A. R., Aird, K. A., et al. 2011, PASP, 123, 568

Chantry, G. W. 1971, Submillimetre Spectroscopy (London: Academic Press)

Colafrancesco S. 2008, MNRAS, 385, 2041

Colafrancesco, S. 2010, in Astrophysics and Cosmology after Gamow: Proceedings of the 4th Gamow International Conference on Astrophysics and Cosmology After Gamow and the 9th Gamow Summer School, Astronomy and Beyond: Astrophysics, Cosmology, Radio Astronomy, High Energy Physics and Astrobiology, AIP Conf. Proc., 1206, 5

Colafrancesco, S., Marchegiani, P., \& Buonanno, R. 2011, A\&A, 527, L1

Conversi, L., Fiadino, P., de Bernardis, P., \& Masi, S. 2010, A\&A, 524, A7

de Bernardis P., Bagliani, D., Bardi, A., et al. 2010, in Proc. of the 12th Marcel Grossman Meeting [arXiv: 1002 . 0867]

Foley, R. J., Andersson, K., Bazin, G., et al. 2011, ApJ, 731, 86

Gong, Y., Cooray, A., Silva, M., et al. 2012, ApJ, 745, 49

Griffin, M., Abergel, A., Ade, P., et al. 2007, Adv. Space Res., 40, 612

Hand, N., Appel, J. W., Battaglia, N., et al. 2011, ApJ, 736, 39

Hincks, A. D., Acquaviva, V., Ade, P. A. R., et al. 2010, ApJS, 191, 423

Holmes, W. A., Bock, J. J., Crill, B. P., et al. 2008, Appl. Opt., 47, 5996

Itoh, N., Kohyama, Y., \& Nozawa, S. 1998, ApJ, 502, 7

Leach, S. M., Cardoso, J. F., Baccigalupi, C., et al. 2008, A\&A, 491, 597

Marriage, T. A., Acquaviva, V., Ade, P. A. R., et al. 2011, ApJ, 737, 61

Masi, S., Ade, P. A. R., Bock, J. J., et al. 2006, A\&A, 458, 687

Masi, S., Battistelli, E., Brienza, D., et al. 2008, Mem. S.A.It., 79, 887

Mather, J. C., Fixen, D. J., \& Shafer, R. A. 1993, Proc. SPIE, 2019, 168

Planck Collaboration 2011a, A\&A, 536, A1

Planck Collaboration 2011b, A\&A, 536, A8

Planck Collaboration 2011c, A\&A, 536, A26

Planck HFI Core Team 2011, A\&A, 536, A4

Rephaeli, Y., Sadeh, S., \& Shimon, M. 2006, Riv. Nuovo Cimento, 29, 1

Sehgal, N., Trac, H., Acquaviva, V., et al. 2011, ApJ, 732, 44

Schwan D., Ade, P. A. R., Basu, K., et al. 2010 [arXiv: 1008. 0342]

Story, K., Aird, K. A., Andersson, K., et al. 2011, ApJ, 735, L36

Sunyaev, R. A., \& Zeldovich, Y. B. 1972, Comm. Ap. Sp. Phys., 4, 173

Swetz, D. S., Ade, P. A. R., Amiri, M., et al. 2011, ApJS, 194, 41

Williamson, R., Benson, B. A., High, F. W., et al. 2011, ApJ, 738, 139 